\title{
DIFFERENTIAL-ALGEBRAIC APPROACH TO CONSTRUCTING REPRESENTATIONS OF COMMUTING DIFFERENTIATIONS IN FUNCTIONAL SPACES AND ITS APPLICATION TO NONLINEAR INTEGRABLE DYNAMICAL SYSTEMS
}

\author{
ANATOLIJ K. PRYKARPATSKI, KAMAL N. SOLTANOV, AND EMIN ÖZÇAĞ
}

\begin{abstract}
There is developed a differential-algebraic approach to studying the representations of commuting differentiations in functional differential rings under nonlinear differential constraints. An example of the differential ideal with the only one conserved quantity is analyzed in detail, the corresponding Lax type representations of differentiations are constructed for an infinite hierarchy of nonlinear dynamical systems of the Burgers and Korteweg-de Vries type. A related infinite bi-Hamiltonian hierarchy of Lax type dynamical systems is constructed.
\end{abstract}

\section{INTRODUCTION}

We consider the ring $\mathcal{K}:=\mathbb{R}\{\{x, t\}\},(x, t) \in \mathbb{R}^{2}$, of convergent germs of real-valued smooth Schwartz type functions from $S\left(\mathbb{R}^{2} ; \mathbb{R}\right)$ and construct the associated differential quotient ring $\mathcal{K}\{u\}:=\operatorname{Quot}(\mathcal{K}[\Theta u])$ with respect to a functional variable $u \in \mathcal{K}$, where $\Theta$ denotes [11, 20, 5, 6, 9] the standard monoid of all commuting differentiations $D_{x}$ and $D_{t}$, satisfying the standard Leibnitz rule, and defined by the natural conditions

$$
D_{x}(x)=1=D_{t}(t), \quad D_{t}(x)=0=D_{x}(t),
$$

The ideal $I\{u\} \subset \mathcal{K}\{u\}$ is called differential if the condition $I\{u\}=\Theta I\{u\}$ holds. In the differential ring $\mathcal{K}\{u\}$ the differentiations

$$
D_{t}, D_{x}: \mathcal{K}\{u\} \rightarrow \mathcal{K}\{u\}
$$

satisfy the algebraic commuting relationship

$$
\left[D_{t}, D_{x}\right]=0 .
$$

For an arbitrarily chosen function $u \in \mathcal{K}$ the only representation of (1.3) in the $\mathcal{K}\{u\}$ is of the form

$$
D_{t}=\partial / \partial t, \quad D_{x}=\partial / \partial t
$$

being the usual partial differentiations. Nonetheless, if the function $u \in \mathcal{K}$ satisfies some additional nonlinear differential-algebraic constraint $Z[u]:=Z\left(u, D_{t} u, D_{x} u, \ldots\right)=0$, imposed on the ring $\mathcal{K}\{u\}$ for some element $Z[u] \in \mathcal{K}\{u\}$, other nontrivial representations of the differentiations (1.2) in the corresponding reduced and invariant differential ring $\overline{\mathcal{K}}\{u\}:=\left.\mathcal{K}\{u\}\right|_{Z[u]=0} \subset \mathcal{K}\{u\}$ can exist.

Below we will consider in detail this situation and construct the corresponding representations of the commuting relationship (1.3), which are polynomially dependent on $u \in \mathcal{K}$ and its derivatives with respect to the differentiation $D_{x}$. The found representations of commuting differentiations $D_{t}$ and $D_{x}$ are interpreted as the corresponding Lax type representations for an infinite hierarchy of nonlinear dynamical systems of Burgers and Korteweg de Vries type.

1991 Mathematics Subject Classification. 35A30, 35G25, 35N10, 37K35, 58J70,58J72, 34A34 .

Key words and phrases. differential rings, differential constraints, representation of differentials, nonlinear vector fields, conserved quantities, Lax type integrability. 
Remark 1.1. There are interesting applications [17, 1] of the problem above in the case when the differentiations $D_{t}$ and $D_{x}: \mathcal{K}\{u\} \rightarrow \mathcal{K}\{u\}$ are defined by means of the Lie algebraic conditions

$$
\left[D_{x}, D_{t}\right]=\left(D_{x} u\right) D_{x}
$$

and

$$
D_{x}(x)=1=D_{t}(t), \quad D_{t}(x)=u, \quad D_{x}(t)=0
$$

for $(x, t) \in \mathbb{R}^{2}$ and $u \in \mathcal{K}$.

The corresponding representations of the Lie algebraic relationships (1.5) and (1.6) under the differential constraints $D_{t}^{N+1} u=0, N \in \mathbb{Z}_{+}$, of Riemann type imposed on the ideal $\mathcal{K}\{u\}$, prove to be finite dimensional, being equivalent to their so called Lax type representations, important for the integrability theory [2, 13, 18, 24, 1] of nonlinear hydrodynamical systems on functional manifolds.

\section{Differential RINGS With The ONLY CONSERVED QUANTITY CONSTRAint}

Let us pose the following problem:

Problem 2.1. To describe the possible representations of the differentiations $D_{t}, D_{x}$, in the ring $\overline{\mathcal{K}}\{u\}=\left.\mathcal{K}\{u\}\right|_{Z[u]=0}$, defined by a constraint $Z[u] \in \mathcal{K}\{u\}, u \in \mathcal{K}$, and satisfying the conditions (1.1) and (1.3).

As is easy to observe, for the case of arbitrarily chosen function $u \in \mathcal{K}$ and the representation of (1.3) in the ring $\mathcal{K}\{u\}$ is given by the unique expressions (1.4). Another situation arises if there is some differential constraint $Z[u]:=Z\left(u, D_{x} u, D_{t} u, \ldots\right)=0, Z[u] \in \mathcal{K}\{u\}$, imposed on the function $u \in \mathcal{K}$. Then one can expect that the commutation condition (1.3), if realized in the constrained ideal $\overline{\mathcal{K}}\{u\}:=\left.\mathcal{K}\{u\}\right|_{Z[u]=0}$, will be much more specified and in some cases the corresponding representations may appear to be even finite dimensional. The latter may by of nontrivial interest for some applications in applied sciences, especially when these imposed constraints possess some interesting physical interpretation. To be further more precise, we need to involve here some additional differential-algebraic preliminaries [6, 7, 8, 9,

Consider the ring $\mathcal{K}\{u\}, u \in \mathcal{K}$, and the exterior differentiation $d: \mathcal{K}\{u\} \rightarrow \Lambda^{1}(\mathcal{K}\{u\}), \ldots$, $d: \Lambda^{p}(\mathcal{K}\{u\}) \rightarrow \Lambda^{p+1}(\mathcal{K}\{u\})$ for $p \in \mathbb{Z}_{+}$, acting in the freely generated Grassmann algebras $\Lambda(\mathcal{K}\{u\})=\oplus_{p \in \mathbb{Z}_{+}} \Lambda^{p}(\mathcal{K}\{u\})$, where by definition,

$$
\begin{aligned}
& \Lambda^{1}(\mathcal{K}\{u\}):=\mathcal{K}\{u\} d x+\mathcal{K}\{u\} d t+\sum_{j, k \in \mathbb{Z}_{+}} \mathcal{K}\{u\} d u^{(j, k)}, \quad u^{(j, k)}:=D_{t}^{j} D_{x}^{k} u, \\
& \Lambda^{2}(\mathcal{K}\{u\}):=\mathcal{K}\{u\} d \Lambda^{1}(\mathcal{K}\{u\}), \ldots, \quad \Lambda^{p+1}(\mathcal{K}\{u\}):=\mathcal{K}\{u\} d \Lambda^{p}(\mathcal{K}\{u\}),
\end{aligned}
$$

The triple $\mathcal{A}:=(\mathcal{K}\{u\}, \Lambda(\mathcal{K}\{u\}), d)$ is called the Grassmann differential algebra [9] with generatrix $u \in \mathcal{K}$. In the algebra $\mathcal{A}$ one naturally defines the action of differentiations $D_{t}, D_{x}$ and $\partial / \partial u^{(j, k)}: \mathcal{A} \rightarrow \mathcal{A}, j, k \in \mathbb{Z}_{+}$, as follows:

$$
\begin{aligned}
D_{t} u^{(j, k)} & =u^{(j+1, k)}, D_{x} u^{(j, k)}=u^{(j, k+1)} \\
D_{t} d u^{(j, k)} & =d u^{(j+1, k)}, D_{x} d u^{(j, k)}=d u^{(j, k+1)} \\
d P[u] & =\sum_{j, k \in \mathbb{Z}_{+}}( \pm) \partial P[u] / \partial u^{(j, k)} \wedge d u^{(j, k)}:=P^{\prime}[u] \wedge d u
\end{aligned}
$$

where the sign " $\wedge$ " denotes the standard [10] exterior multiplication in the differential Grassmann algebra $\Lambda(\mathcal{K}\{u\})$, and for any $P \in \Lambda(\mathcal{K}\{u\})$ the mapping

$$
P^{\prime}[u] \wedge: \Lambda(\mathcal{K}\{u\}) \rightarrow \Lambda(\mathcal{K}\{u\})
$$

is a linear differential operator in $\Lambda(\mathcal{K}\{u\})$. The following commutation properties

$$
D_{x} d=d D_{x}, \quad D_{t} d=d D_{t}
$$

hold in the Grassmann differential algebra $\mathcal{A}$. The following remark 9 is also important. 
Remark 2.2. The Lie derivative $L_{V}: \mathcal{K}\{u\} \rightarrow \mathcal{K}\{u\}$ with respect to a vector field $V: \mathcal{K}\{u\} \rightarrow$ $T(\mathcal{K}\{u\})$, satisfying the condition $L_{V}: \mathcal{K} \subset \mathcal{K}$, can be uniquely extended to the differentiation $L_{V}: \mathcal{A} \rightarrow \mathcal{A}$, satisfying the commutation condition $L_{V} d=d L_{V}$.

The variational derivative, or the functional gradient $\nabla P[u] \wedge \in \Lambda(\mathcal{K}\{u\})$, is defined for any $P \in \Lambda(\mathcal{K}\{u\})$ by means of the following expression:

$$
\nabla P[u] \wedge:=\sum_{j, k \in \mathbb{Z}_{+}}(-1)^{ \pm}\left(-D_{t}\right)^{j}\left(-D_{x}\right)^{k}\left(\partial P[u] / \partial u^{(j, k)}\right) \wedge,
$$

which can be equivalently rewritten as

$$
\nabla P[u] \wedge=P^{\prime}[u]^{*} \wedge
$$

where a mapping $P^{\prime}[u]^{*} \wedge: \Gamma(\mathcal{K}\{u\}) \rightarrow \Gamma(\mathcal{K}\{u\})$ is formally adjoint to that of (2.3) and is naturally defined on the space $\Gamma(\mathcal{K}\{u\})$ of vector fields on $\mathcal{K}\{u\}$. The latter is strongly based on the following important lemma, stated for a special case in [9, 5, 6, 17, 8, 14.

Lemma 2.3. Let the differentiations $D_{x}$ and $D_{t}: \Lambda(\mathcal{K}\{u\}) \rightarrow \Lambda(\mathcal{K}\{u\})$ satisfy the conditions (2.2). Then the relation

$$
\begin{gathered}
\operatorname{Ker} \nabla /(\operatorname{Im} d \oplus \mathbb{R}) \simeq H^{1}(\Lambda(\mathcal{K}\{u\})):= \\
=\operatorname{Ker}\left\{d: \Lambda^{1}(\mathcal{K}\{u\}) \rightarrow \Lambda^{2}(\mathcal{K}\{u\})\right\} / d \Lambda^{0}(\mathcal{K}\{u\})
\end{gathered}
$$

is a canonical isomorphism, where $H^{1}(\mathcal{A})$ is the corresponding cohomology classes of the Grassmann complex $\Lambda(\mathcal{K}\{u\})$.

It is well known that in the case of the differential ring $\mathcal{K}\{u\}$ all of the cohomology classes $H^{j}(\mathcal{A}), j \in \mathbb{Z}_{+}$, are trivial, giving rise to the well known classical relationship $\operatorname{Ker} \nabla=\operatorname{Im} D_{x} \oplus \operatorname{Im} D_{t} \oplus \mathbb{R}$. In addition, the following simple relationship holds:

$$
\nabla \cdot\left(\operatorname{Im} D_{x} \oplus \operatorname{Im} D_{t}\right)=0 .
$$

Based on Lemma 2.3 one can define the equivalence class $\widetilde{\mathcal{A}}:=\mathcal{A} /\left\{\operatorname{Im} D_{x} \oplus \operatorname{Im} D_{t} \oplus \mathbb{R}\right\}$ $:=\mathcal{D}(\mathcal{A} ; d x d t)$, whose elements will be called functionals, that is any element $\gamma \in \mathcal{D}(\mathcal{A} ; d x d t)$ can be represented as a suitably defined integral $\gamma:=\iint d x d t \gamma[u] \in \mathcal{D}(\mathcal{A} ; d x d t)$ for some $\gamma[u] \in \Lambda(\mathcal{K}\{u\})$ with respect to the Lebesgue measure $d x d t$ on $\mathbb{R}^{2}$.

Proceed now to treating the case when the following simple enough and uniform in the variables $(x, t) \in \mathbb{R}^{2}$ differential constraint is imposed on a function $u \in \mathcal{K}$ generating the ideal $\mathcal{K}\{u\}$ :

$$
D_{t} u+K[u]=0
$$

where a smooth element $K[u]:=D_{x} k[u]$ for some element $k[u] \in \overline{\mathcal{K}}\{u\}$ is defined by means of the following differential-algebraic condition: except the relationship (2.9), that is

$$
D_{t} u+D_{x} k[u]=0,
$$

there exists no other additional constraint of the form

$$
D_{t} h[u]+D_{x} H[u]=0
$$

for some elements $h[u], H[u] \in \overline{\mathcal{A}}$ from the reduced Grassmann differential algebra $\overline{\mathcal{A}}:=$ $(\bar{K}\{u\}, \Lambda(\bar{K}\{u\}), d)$.

Remark 2.4. It is necessary to mention that additional conservation relationships like (2.11) can exist in an extended differential algebra $\tilde{\mathcal{A}}:=(\tilde{K}\{u\}, \Lambda(\tilde{K}\{u\}), d)$, where $\tilde{K}\{u\}:=$ $\bar{K}\{u ; \alpha\}$ for some additional set of elements $\alpha \subset \mathcal{K}$.

Based on the differential-algebraic setting, described above, the latter condition can be interpreted naturally in the following way: if to define the spaces of functionals $\mathcal{D}(\overline{\mathcal{A}} ; d x):=$ $\overline{\mathcal{A}} / D_{x} \overline{\mathcal{A}}$ and $\mathcal{D}(\overline{\mathcal{A}} ; d t)=\overline{\mathcal{A}} / D_{t} \overline{\mathcal{A}}$ on the reduced Grassmann differential algebra $\overline{\mathcal{A}}$, then from the functional point of view these factor spaces $\mathcal{D}(\overline{\mathcal{A}} ; d x)$ and $\mathcal{D}(\overline{\mathcal{A}} ; d t)$ can be understood more classically as the corresponding spaces of suitably defined integral expressions subject to the measures $d x$ and $d t$, respectively. Then the relationship (2.11) means equivalently that the functional $h:=\int d x h[u] \in \mathcal{D}(\overline{\mathcal{A}} ; d x)$ is a conserved quantity for the differentiation $D_{t}$, and the functional $H:=\int d t H[u] \in \mathcal{D}(\overline{\mathcal{A}} ; d t)$ is a conserved quantity for the differentiation $D_{x}$. 
Since the differential relationship (2.9) naturally defines on the reduced ring $\overline{\mathcal{K}}\{u\}$ the vector field $K: \overline{\mathcal{K}}\{u\} \rightarrow T(\overline{\mathcal{K}}\{u\})$, one can construct the corresponding Lie derivative $L_{K}: \overline{\mathcal{A}} \rightarrow \overline{\mathcal{A}}$ along this vector field and calculate the quantity $L_{K} \varphi[u] \in \Lambda(\overline{\mathcal{K}}\{u\})$, $\varphi[u]:=\nabla \gamma[u] \wedge \in \Lambda(\overline{\mathcal{K}}\{u\})$ for any conserved quantity $\gamma \in \mathcal{D}(\overline{\mathcal{A}} ; d x)$ with respect to the differentiation $D_{t}$. The following classical lemma [12, 14, 1, 18, 14, holds.

Lemma 2.5. (E. Noether, P.Lax) Let a quantity $\varphi[u] d u \in \Lambda^{1}(\overline{\mathcal{K}}\{u\})$ be such that the following conditions:

$$
D_{t} \varphi[u]+K^{\prime, *}[u] \varphi[u]=0, \varphi^{\prime, *}[u]=\varphi^{\prime}[u],
$$

hold for any $u \in \mathcal{K}$, satisfying the differential constraint (2.9). Then the functional

$$
\gamma:=\int_{0}^{1} d \lambda \int d x \varphi[\lambda u] u \in \mathcal{D}(\overline{\mathcal{A}} ; d x)
$$

is a conserved quantity with respect to the differentiation $D_{t}$.

As a simple corollary from the Lemma 2.5, subject to the problem posed above, we need to show that the only solution to the equation (2.12) is the trivial element $\varphi[u]=1 \in \Lambda^{0}(\overline{\mathcal{K}}\{u\})$. To solve this problem effectively enough (but not in a most general form) we will take into account [3, 21] that the linear equation (2.12) possesses a partial asymptotical solution with respect to the parameter $\lambda \in \mathbb{R}$ as $|\lambda| \rightarrow \infty$, such that the element

$$
\varphi[u] \sim \psi[u ; \lambda] \exp \left\{-\lambda^{n} t-\lambda x+\partial^{-1} u\right\}
$$

for any $n \in \mathbb{Z}_{+} \backslash\{0,1\}$ belongs to the finally extended differential algebra $\Lambda^{0}(\tilde{\mathcal{K}}\{u\}) \simeq$ $\Lambda^{0}\left(\overline{\mathcal{K}}\left\{u ; \partial^{-1} u\right\}\right)$ by means of the element $\partial^{-1} u \in \mathcal{K}$. Here, by definition, $D_{x} \cdot \partial^{-1}=\mathbf{1}$ : $\Lambda(\overline{\mathcal{K}}\{u\}) \rightarrow \Lambda(\overline{\mathcal{K}}\{u\})$ is the identity mapping and, by construction, the element $\psi[u ; \lambda] \in$ $\Lambda^{0}(\overline{\mathcal{K}}\{u\})$ and satisfies the limiting condition $\lim _{|\lambda| \rightarrow \infty} \psi[u ; \lambda]:=1$. It is easy to observe that the expression (2.14) guarantees that the only conservative quantity with respect to the differentiation $D_{t}$ is the functional $\gamma_{0}=\int d x u \in \mathcal{D}(\overline{\mathcal{A}} ; d x)$. Having taken into account the condition that the expression $\varphi[u ; \lambda]=1$ is the only solution to the equation (2.12) for all $u \in \mathcal{K}$, satisfying the constraint (2.9), one easily finds from (2.14) that

$$
u=\lambda-D_{x} \ln \psi[u ; \lambda]
$$

for any $\lambda \in \mathbb{R}$ as $|\lambda| \rightarrow \infty$. If now to define the expression

$$
v[u ; \lambda]:=\psi[u ; \lambda] \exp \left(-\lambda x-\lambda^{n} t\right) \in \Lambda^{0}(\overline{\mathcal{K}}\{u\}),
$$

such that

$$
u=-D_{x} \ln v[u ; \lambda]
$$

one can easily see from the constraint (2.9) and the equation (2.12) that the functional $\gamma_{0}=\int d x u \in \mathcal{D}(\overline{\mathcal{A}} ; d x)$ will be the only conserved quantity for the differentiation $D_{t}$ in the differential algebra $\overline{\mathcal{A}}$, if the element $v[u ; \lambda] \in \Lambda^{0}(\overline{\mathcal{K}}\{u\})$ satisfies a necessarily linear differential relationship

$$
D_{t} v[u ; \lambda]+A\left(D_{x}\right) v[u ; \lambda]=0
$$

for some linear differential mapping $A\left(D_{x}\right): \Lambda^{0}(\overline{\mathcal{K}}\{u\}) \rightarrow \Lambda^{0}(\overline{\mathcal{K}}\{u\})$, not depending on the element $u \in \mathcal{K}$. Otherwise, it would then provide with an additional constraint on the function $u \in \mathcal{K}$, giving rise to an additional conserved quantity for the differentiation $D_{t}$ in the reduced differential algebra $\Lambda(\overline{\mathcal{K}}\{u\})$. Taking this reasoning into account, we can make the shift $\mathcal{K} \ni u \rightarrow \xi u \in \mathcal{K}, \xi \in \mathbb{R}$, and consider the relationship (2.18) as $\xi \rightarrow 0$. As a result one obtains that the limiting function $\bar{v}[x, t ; \lambda]:=\exp \left(-\lambda x-\lambda^{n} t\right) \in \Lambda^{0}(\overline{\mathcal{K}}\{0\})$ satisfies the linear differential relationship

$$
D_{t} \bar{v}[x, t ; \lambda]+K^{\prime, *}[0] \bar{v}[x, t ; \lambda]=0,
$$

whence one derives easily that

$$
K^{\prime}[0]=-D_{x}^{n}
$$

for the before chosen integer $n \in \mathbb{Z}_{+} \backslash\{0,1\}$. Thus, from (2.19) one brings about that the following linear relationship

$$
D_{t} v[u ; \lambda]+\left(-D_{x}\right)^{n} v[u ; \lambda]=0
$$


holds for any $u \in \mathcal{K}$, satisfying the constraint (2.9).

Turning now back to the relationship (2.17), one can easily obtain that the function $u \in \mathcal{K}$ under our constraint (2.9) guarantees that the only conserved quantity with respect to the differentiation $D_{t}$ is the functional $\gamma_{0}=\int d x u \in \mathcal{D}(\overline{\mathcal{A}} ; d x)$ and satisfies the following nonlinear differential relationships:

$$
D_{t_{n}} u+K_{n}[u]=0
$$

with respect to the evolution parameters $t_{n} \in \mathbb{R}$ for any $n \in \mathbb{Z}_{+} \backslash\{0,1\}$, where the corresponding vector fields $K_{n}: \overline{\mathcal{K}}\{u\} \rightarrow \Gamma(\overline{\mathcal{K}}\{u\})$ are equal to the analytical expressions

$$
K_{n}[u]=\exp \left(\partial^{-1} u\right) \sum_{k=0}^{n-1} \frac{n !}{(n-k) ! k !}\left(D_{x}^{n-k} u\right) D_{x}^{k} \exp \left(-\partial^{-1} u\right)
$$

of the Burgers and Korteweg-de Vries type. If to recall that $K_{n}[u]=D_{x} k_{n}[u]$, then one easily finds from (2.23) that the following recurrent relationship

$$
k_{n+1}[u]=-u k_{n}[u]+D_{x} k_{n}[u]
$$

holds for $n \in \mathbb{N}, k_{1}[u]:=u \in \mathcal{K}$. For the simplest cases one obtains from (2.24) the constraints

$$
\begin{gathered}
n=2: \quad D_{t_{2}} u-D_{x}^{2} u+2 u D_{x} u=0, \\
n=3: \quad D_{t_{3}} u-D_{x}^{3} u+3 D_{x}\left(u D_{x} u\right)-D_{x}\left(u^{3}\right)=0
\end{gathered}
$$

and so on. In addition to the constraint (2.22) for $n \in \mathbb{N}$, from (2.15) one obtains the following interesting relationship on the element $\psi[u ; \lambda] \in \Lambda^{0}(\overline{\mathcal{K}}\{u\})$ :

$$
D_{x} \psi=(\lambda-u) \psi
$$

for any $\lambda \in \mathbb{R}$. Having taken into account the condition (2.21), we also easily find that the linear differentiation expression

$$
D_{t_{n}} \psi=\sum_{k=0}^{n} \frac{n !}{(n-k) ! k !}(-\lambda)^{n-k} D_{x}^{k-1}[(\lambda-u) \psi]
$$

holds for $n \in \mathbb{N}$ and any $\lambda \in \mathbb{R}$, which together with the expression (2.27) is equivalent to the related Lax type representations for the vector fields (2.23). Thus, one can formulate the following theorem.

Theorem 2.6. The scalar multiplicative expressions (2.27) and (2.28) are the corresponding representations of the commuting differential relationship (1.3) in the differential algebra $\Lambda^{0}(\overline{\mathcal{K}}\{u\}) \simeq \overline{\mathcal{K}}\{u\}$ under the constraints (2.22), being equivalent to the related Lax type representations for the vector fields (2.23).

Concerning the case $n=3$ some related preliminary results, including the representations (2.27) and (2.28), were before obtained in the work [19, 22. In addition, in 19 there was stated that the odd members of dynamical systems (2.28) possess an infinite hierarchy of conserved nonlocal quantities and two compatible 2, 14, 18 Poisson structures $\vartheta, \eta$ : $T^{*}(\overline{\mathcal{K}}\{u\}) \rightarrow T(\overline{\mathcal{K}}\{u\})$ subject to the extended ring $\tilde{\mathcal{K}}\{u\}=\overline{\mathcal{K}}\left\{u ; \partial^{-1} u\right\}$ and equal to the expressions

$$
\begin{aligned}
\vartheta & =\partial \exp \left(\partial^{-1} u\right) \partial \exp \left(\partial^{-1} u\right) \partial \\
\eta & =\partial \exp \left(\partial^{-1} u\right) \partial^{3} \exp \left(\partial^{-1} u\right) \partial .
\end{aligned}
$$

With respect to the structures (2.29) the dynamical system (2.26) is representable as biHamiltonian flow in the ring $\overline{\mathcal{K}}\{u\}$ :

$$
d u / d t_{3}=-\vartheta \operatorname{grad} H_{3}=-\eta \operatorname{grad} \bar{H}_{3},
$$

where

$$
H_{3}:=\frac{1}{2} \int u^{2} \exp \left(-2 \partial^{-1} u\right) d x, \quad \bar{H}_{3}:=\frac{1}{2} \int \exp \left(-2 \partial^{-1} u\right) d x .
$$

Similar results can be, evidently, stated for any constraint of (2.22). 
It is also easy to observe that the recurrent relationship (2.24) can be rewritten by means of the recursion operator

$$
\Lambda=\partial-\partial u \partial^{-1}
$$

for the corresponding vector fields (2.23), as

$$
K_{n+1}[u]=\Lambda K_{n}[u],
$$

$n \in \mathbb{N}$. Moreover, as follows from (2.30), the next interesting algebraic relationship

$$
\Lambda^{2}=\eta \vartheta^{-1}=\partial \exp \left(\partial^{-1} u\right) \partial^{2} \exp \left(-\partial^{-1} u\right) \partial^{-1}
$$

holds.

Corollary 2.7. As a simple corollary from the recurrent relationship (2.33) one obtains that all of the vector fields (2.23) are commuting [2, 18] to each other:

$$
\left[K_{n}, K_{m}\right]=0
$$

for any $n, m \in \mathbb{N}$.

The result above also easily follows from the differential relationship (2.21). Really, since its solutions for different $n \in \mathbb{Z}_{+} \backslash\{0,1\}$ depend only on the initial conditions $\left.u\right|_{t_{n}=0} \in \mathcal{K}\{u\}$, the corresponding differentiations

$$
D_{t_{n}}=\exp \left[(-1)^{n+1} D_{x}^{n}\right]
$$

being well defined on the linear Schwartz space $S(\mathbb{R} ; \mathbb{R})$, are a priori commuting to each other, that is

$$
\left[D_{t_{n}}, D_{t_{m}}\right]=0 \text {. }
$$

The latter is equaivalent to the commutation condition (2.35).

\section{Conclusion}

The linear differential relationships (2.27) and (2.28) in the differential Grassmann algebra $\Lambda^{0}(\overline{\mathcal{K}}\{u\})$ for $u \in \mathcal{K}$, satisfying the differential constraint (2.9), realize scalar representations of the basic differentiations $D_{t}$ and $D_{x}$, a priori commuting to each other on the whole functional manifold of solutions to differential constraints (2.22). Simultaneously, they are equivalent within the well known integrability theory interpretation as the related LaxZakharov-Shabat type representation [13] for the vector fields (2.23), useful in many cases for practical applications. It is also evident that a similar approach can be applied to other cases, when there are imposed on the ideal $\mathcal{K}\{u, v, \ldots\}$, depending on two or more different functions $u, v, \ldots \in \mathcal{K}$, some a priori given constraint $0=Z[u] \in \mathcal{K}\{u, v, \ldots\}$. In this case one can also try to construct the corresponding finite dimensional representations of differentiations $D_{t}$ and $D_{x}$ in $\mathcal{K}\{u, v, \ldots\}$. For instance, the following two-component hydrodynamic type system

$$
\left.\begin{array}{c}
u_{t}=u_{x x}+2 u u_{x}+v_{x} \\
v_{t}=u_{x} v+u v_{x}
\end{array}\right\}:=K[u, v],
$$

can be realized as a constraint imposed on the functional ring $\mathcal{K}\{u, v\}$. It was singled out in 23 as suspicious on the Lax type integrability. Based on the techniques, devised in [19, 17, [15] and in the present work [16], we were able to prove [4] the Lax type integrability of (3.1) and to present an infinite hierarchy of new Lax type integrable dynamical systyems of the hydrodynamic type.

\section{Acknowledgements}

Authors are gratefully acknowledge partial support of the research in this paper from the Turkey-Ukrainian: TUBITAK-NASU Grant 110T558. A.K. Prykarpatski cordially thanks Prof. A. Augustynowicz (Gdansk University, Poland) and Prof. M.V. Pavlov (Lomonosov Sate University, Russian Federation) for useful discussions of the results obtained. Special thanks belong to Referee for many usefull remarks, comments and professional suggestions which made a manuscript both more clear and readable. 


\section{REFERENCES}

[1] Blackmore D., Prykarpatsky A.K. and Samoylenko V.H., Nonlinear dynamical systems of mathematical physics. World Scientific Publisher, NJ, USA, 2011

[2] Blaszak M., Bi-Hamiltonian dynamical systems. NY, Springer, 1998

[3] Coddington E.A., Levinson N., Theory of differential equations. New York, McGraw-Hill, 1955

[4] Blackmore Denis, Prykarpatski Anatolij K., Özçağ Emin and Soltanov Kamal. The Lax integrability of a two component hierarchy of the Burgers type dynamical systems within asymptotic and differentialalgebraic approaches. arXiv:submit/0827860 [nlin.SI] 21 Oct 2013

[5] Gelfand I.M. and Dikiy L.A., Integrable nonlinear equations and Liouville theorem, Function. Anal. Appl. 13 (1979), 8-20.

[6] Gelfand I.M. and Dikiy L.A., The calculus of jets and nonlinear Hamiltonian systems, Function. Anal. Appl., April--June, 1978, Volume 12, Issue 2, pp 81-94

[7] Gelfand I.M. and Dikiy L.A., A Lie algebra structure in a formal variational calculation. Function. Anal. Appl., January-March, 1976, Volume 10, Issue 1, pp 16-22

[8] Gelfand I.M. and Dikiy L.A., The resolvent and Hamiltonian systems. Function. Anal. Appl.., AprilJune, 1977, Volume 11, Issue 2, pp 93-105

[9] Gelfand I.M., Manin Yu.I. and Dikiy L.A., Poisson brackets and the kernel of the variational derivative in the formal calculus of variations. Function. Anal. Appl., October-December, 1976, Volume 10, Issue 4, pp 274-278

[10] Godbillon C., Geometri differentielle et mecanique analytique. Hermann, Paris, 1969

[11] Kaplanski I., Introduction to differential algebra. NY, 1957

[12] Lax Peter D., Almost Periodic Solutions of the KdV Equation. Source: SIAM Review, Vol. 18, No. 3 (Jul., 1976), pp. 351-375

[13] Novikov S.P. (Editor), Theory of Solitons: The Inverse Scattering Method. Springer, 1984

[14] Olver P., Applications of Lie Groups to Differential Equations, Second Edition, Springer-Verlag, New York, 1993

[15] Prykarpatsky Y.A. Finite dimnesnional local and nonlocal reductions of one type of hydrodynamic systems. Reports Math Phys, 50, N3 (2002) p. 349-360

[16] Prykarpatski Anatolij K., Özçă̆ Emin and Soltanov Kamal, Differential-algebraic approach to constructing representations of commuting differentiations in functional spaces and its application to nonlinear integrable dynamical systems. arXiv:submit/0729174 [nlin.SI] 1 Jun 2013

[17] Prykarpatsky A.K., Artemovych O.D., Popowicz Z. and Pavlov M.V., Differential-algebraic integrability analysis of the generalized Riemann type and Korteweg-de Vries hydrodynamical equations. J. Phys. A: Math. Theor. 43 (2010) 295205 (13pp)

[18] Prykarpatsky A. and Mykytyuk I., Algebraic integrability of nonlinear dynamical systems on manifolds: classical and quantum aspects. Kluwer Academic Publishers, the Netherlands, 1998

[19] Prytula M.M., Prykarpatski A.K. and Vovk M.I., On the complete integrability and liniarization of a Burgers-Korteweg de vries equation. Journal of Mathematical Sciences, Vol. 161, No. 1, p. 1-6, 2009

[20] Ritt J.F., Differential algebra. AMS-Colloqium Publications, vol. XXXIII, New York, NY, Dover Publ., 1966

[21] Shubin M., Lectures on mathematical physics. Moscow State University, Moscow, Russian Federation

[22] Tasso H. Hamiltonian formulation of odd Burgers hierarchy. J.Phys. Math.Gen., 29, 1996, p. 7779-7784

[23] Tsuchida T. and Wolf T. Classification of polynomial integrable systems of mixed scalar and vector evolution equations. I. J. Phys. A: Math.Gen., 38,2005, p. 7691-7733

[24] Wilson G., On the quasi-Hamiltonian formalism of the KdV equation. Physics Letters, 132(8/9) (1988), p. $445-450$

The Department of Applied Mathematics at AGH University of Science and Technology of KRAKOW, POLAND

E-mail address: pryk.anat@ua.fm, prykanat@cybergal.com

E-mail address: sultan_kamal@hotmail.com, soltanov@hacettepe.edu.tr

E-mail address: ozcag1@hacettepe.edu.tr

The Department of Mathematics at the Hacettepe University of Ankara, Turkey 